\title{
Applying network analysis and Nebula (neighbor-edges based and unbiased leverage algorithm) to ToxCast data
}

\author{
Hao Ye, Heng Luo, Hui Wen Ng, Joe Meehan, Weigong Ge, Weida Tong, Huixiao Hong* \\ Division of Bioinformatics and Biostatistics, National Center for Toxicological Research, U.S. Food \\ and Drug Administration, 3900 NCTR Road, Jefferson, AR 72079, USA \\ *Correspondence should be addressed to Dr. Huixiao Hong at huixiao.hong@fda.hhs.gov
}

The findings and conclusions in this article have not been formally disseminated by the US Food and Drug Administration (FDA) and should not be construed to represent the FDA determination or policy. 


\begin{abstract}
Background: ToxCast data have been used to develop models for predicting in vivo toxicity. To predict the in vivo toxicity of a new chemical using a ToxCast data based model, its ToxCast bioactivity data are needed but not normally available. The capability of predicting ToxCast bioactivity data is necessary to fully utilize ToxCast data in the risk assessment of chemicals.

Objectives: We aimed to understand and elucidate the relationships between the chemicals and bioactivity data of the assays in ToxCast and to develop a network analysis based method for predicting ToxCast bioactivity data.

Methods: We conducted modularity analysis on a quantitative network constructed from ToxCast data to explore the relationships between the assays and chemicals. We further developed Nebula (neighbor-edges based and unbiased leverage algorithm) for predicting ToxCast bioactivity data.

Results: Modularity analysis on the network constructed from ToxCast data yielded seven modules. Assays and chemicals in the seven modules were distinct. Leave-one-out cross-validation yielded a $\mathrm{Q}^{2}$ of 0.5416 , indicating ToxCast bioactivity data can be predicted by Nebula. Prediction domain analysis showed some types of ToxCast assay data could be more reliably predicted by Nebula than others.

Conclusions: Network analysis is a promising approach to understand ToxCast data. Nebula is an effective algorithm for predicting ToxCast bioactivity data, helping fully utilize ToxCast data in the risk assessment of chemicals.
\end{abstract}

Keywords: ToxCast; environmental toxicity; network algorithm; Nebula 


\section{Introduction}

Environmental chemicals are found in the home, workplace and other surroundings, thus humans and animals are exposed to them on a daily basis through air, water, food, soil, etc. Currently, about 85,000 chemicals have been registered for use in the US under the Toxic Substances Control Act (TSCA) (TSCA 2015). In addition, 500 1,000 new chemicals are introduced into the environment each year (ATSDR 2014). Approximately, 30,000 chemicals are considered to be widely used in commerce (>1 t/y) (Muir and Howard 2006). However, federal agencies have the capacity to evaluate only a small part of them, and the toxicity of the majority of environmental chemicals is unknown. According to the latest US national report on human exposure to environmental chemicals by US Center for Disease Control (CDC) (released on Feb., 2015), the toxicity data for human exposure are well recorded for only 265 chemicals (CDC 2015). Even in animal tests, only a small fraction of these commonly used chemicals has undergone rigorous safety evaluation due to the high expense. There is great concern regarding the risk of environmental chemicals due to not enough toxicity data. For example, DDT (dichlorodiphenyltrichloroethane) was once viewed as a successful agricultural insecticide and its discoverer was awarded a Nobel Prize in Physiology or Medicine in 1948. However, DDT was later found to cause cancer and its agricultural use was determined to be a threat to wildlife, particularly for birds (Carson 1962). Therefore, DDT has been banned for use in the United States.

The ToxCast project was launched by the US EPA (Dix et al., 2007; Kavlock et al., 2012) with the aim of rapidly screening and facilitating the prioritization of environmental chemicals for further toxicological testing. ToxCast utilized high-throughput screening assays to detect the biological activity of living cells or isolated proteins for some 800 environmental chemicals. Since ToxCast first released the data in 2009, the data have been used to explore the molecular mechanisms or Adverse Outcome Pathways (AOPs) of chemical toxicity. Shah (Shah and Greene 2013) found that drugs with hepatotoxicity showed positive activity on PXR, NRF2, PPARG, according to the bioactivity difference between failed drugs and other environmental chemicals in ToxCast data. Berg (Berg et al., 2013) and Kleinstreuer (Kleinstreuer et al., 2014) utilized chemical-response signatures on human cell systems in ToxCast with the support vector machine (SVM) method to classify toxic and therapeutic mechanisms within 28 mechanism classes. Zang (Zang et al., 2013) identified several structural features that are most relevant to estrogen receptor (ER) activity based on ToxCast data using quantitative structure-activity relationship (QSAR) and machine learning methods. Kleinstreuer (Kleinstreuer et al., 2011) identified developmental toxicity pathways from a subset of ToxCast chemicals using human embryonic stem cells and metabolomics.

There have been many efforts to build models for predicting in vivo animal toxicity based on ToxCast data. Martin (Martin et al., 2011) developed a linear discriminant analysis model for predicting rat reproductive toxicity based on the 309 chemicals of ToxCast phase 1 data. Kleinstreuer (Kleinstreuer et al., 2012) proposed a cancer hazard model for predicting rodent carcinogenicity based on 292 chemicals, 672 assays (mapping to 455 genes) in ToxCast. Sipes (Sipes et al., 2011) constructed a predictive model for predicting in vivo prenatal developmental toxicity in ToxRefDB using ToxCast high-throughput screening data. Liu (Liu et al., 2015) developed supervised machine learning models to predict chronic hepatotoxicity endpoints using the ToxCast bioactivity data, chemical structure descriptors and the combination of both. Abdelaziz (Abdelaziz et al., 2015) developed an online platform iPrior, which could predict 61 toxicity endpoints using multiple descriptor packages and hundreds of in vitro assays.

ToxCast data have been demonstrated to be a reliable and valuable resource for exploring mechanisms of chemical toxicity and for predicting in vivo animal toxicity. In real practice, in order to predict in vivo animal toxicity for new chemicals using predictive models developed based on ToxCast assays data, the ToxCast assays activity data for the new chemicals have to be known first. However, experimentally measuring ToxCast assays activity is time consuming and expensive, hindering the utilization of ToxCast data based predictive models. Therefore, in silico methods for predicting ToxCast bioactivity data of new chemicals are urgently needed to fully realize the utility of ToxCast data. In this study, we developed a novel in silico method based on network analysis, 
Neighbor-Edges Based and Unbiased Leverage Algorithm (Nebula), for predicting ToxCast bioactivity data. We also conducted modularity analysis to elucidate the relationships among the chemicals and assays of ToxCast.

\section{Materials and methods}

\subsection{Study design}

The overall workflow of this study is shown in Figure 1. The chemicals-assays network for ToxCast bioactivity data was constructed first. The Louvain method (Blondel et al., 2008) in the python package "community" was then utilized to decompose the network into multiple densely connected modules based on topological properties. Thereafter, comparative analysis was conducted to explore the characteristics of chemicals and assays in each of the modules. Lastly, Nebula was developed for predicting ToxCast bioactivity data and the performance of Nebula was evaluated using Leave-One-Out (LOO) cross validations.

\subsection{ToxCast data}

The original ToxCast bioactivity data as well as the assay descriptions were downloaded from the US EPA website (http://www.epa.gov/ncct/toxcast/data.html). The bioactivity data ( $\mathrm{AC}_{50}$ values) were transformed to $-\log \mathrm{AC}_{50}$. The terms 'gene symbol', 'tissue', 'biological_process_target' and 'ASSAY_TARGET_FAMILY' in the assay descriptions were used in the enrichment analysis. Also, the chemical name and SMILES code in the chemical annotation files were extracted.

\subsection{Network modularity analysis}

Modularity (Newman 2012) is a parameter between $(0,1)$ used to indicate the amount of signal and to measure the strength of dividing a network into densely connected modules. For a given division of a network into modules, the modularity Q is calculated using formula (1).

$$
Q=\sum_{i=1}^{q}\left[e_{i i}-\left(\sum_{j i} e_{i j}\right)^{2}\right]
$$

Where $r$ is the number of modules,$e_{\mathrm{ii}}$ is the fraction of chemical-assay data with both chemical and assay located within module $i ; e_{\mathrm{i} j} j$ is the fraction of chemical-assay data with the chemical and assay located in two different modules $i$ and $j$. Modularity analysis is the maximization of $\mathrm{Q}$, the process that finds the division to yield the maximum or close to the maximum $\mathrm{Q}$.

\subsection{Permutation tests}

In order to test whether the modularity indicates a signal due to the topological property of the network of ToxCast bioactivity data or could be obtained by chance statistically, two types of permutation tests were conducted to calculate modularity values of random networks. In the first type of permutation, the same ToxCast bioactivity data were randomly assigned to chemicals and assays. Therefore, the degrees (number of data) of chemicals and assays in the random networks may be different from those in the ToxCast bioactivity network. For the second type of permutation, the ToxCast data were randomly assigned the chemical-assay pairs; thus the degrees of chemicals and assays remained the same.

\subsection{Assay description terms enrichment analysis}

Assay description term enrichment in each module was conducted by comparing assays with a description term to assays without the term. Taking module $x$ and assay description term $y$ as an example, we built a $2 \times 2$ contingency table first: 


\begin{tabular}{|l|l|l|l|}
\hline & Assays in module $x$ & Assays not in module $x$ & Total \\
\hline Assays in Term $y$ & $a$ & $c$ & $a+c$ \\
\hline Assays not in Term $y$ & $b$ & $d$ & $b+d$ \\
\hline Total & $a+b$ & $c+d$ & \\
\hline
\end{tabular}

Where $a$ is the number of assays in module $x$ which are described by term $y ; b$ is the number of assays in module $x$ but are not described in term $y$; $c$ indicates the number of assays described by term $y$ but not in module $x ; d$ is the number of assays not in module $x$ and not described by term $y$. From the contingency table, a p value is calculated using Fisher exact test. The enrichment of assays with description term $\mathrm{y}$ in module $\mathrm{x}$ is measured by enrichment factor (EF) that is calculated using formula (2).

$$
\mathrm{EF}=\frac{a}{a+b} / \frac{c}{a+d}
$$

In the enrichment analysis, EF $>=2$ and $\mathrm{p}$ value $<=0.05$ were used to select the assays as enriched in the modules. The assay description terms used in the enrichment analysis are sample tissue, assay target family and biological process target.

\subsection{Pathway enrichment analysis}

A gene list was first generated by extracting the gene symbol in the assay description term 'gene symbol' of the assays included in a module. The gene list was then submitted to the Database for Annotation, Visualization and Integrated Discovery (DAVID) (Huang da et al., 2009) (https://david.ncifcrf.gov/summary.jsp) for pathway enrichment analysis. DAVID provides a comprehensive set of functional annotation tools to understand biological meaning behind a large list of genes. More specifically, the Kyoto Encyclopedia of Genes and Genomes (KEGG) (Kanehisa et al., 2011) pathway enrichment analysis in DAVID that is based on fisher exact test was utilized in this study. At last, $\mathrm{p}$ value $<=0.05$ was used to select the enriched pathways for each module.

\subsection{Chemical difference in the modules}

We first retrieved the SMILES code for the chemicals in the ToxCast data. The online SMILES translator and structure file generator (http://cactus.nci.nih.gov/translate/) developed by NCI was then used to generated twodimensional structures for the chemicals as an output SDF file. The SDF file, was input in Mold $^{2}$ (Hong et al., 2008) for calculating the 777 molecular descriptors for each of the chemicals in ToxCast. Mold ${ }^{2}$ is a software package developed in our laboratory for calculation of molecular descriptors from two-dimensional chemical structures. This software is publicly available (http://www.fda.gov/ScienceResearch/BioinformaticsTools/Mold2/). It is extremely fast because it utilizes a rapid algorithm for recognition of the rings in a chemical structure (Hong \& Xin, 1992a) and uses an efficient coding system for chemical structure representation (Hong \& Xin, 1992b; Hong \& Xin, 1994a) that has been shown to be efficient in the chemical structure elucidation system (Hong \& Xin, 1990; Hong \& Xin, 1994b; Hong et al., 1995; Masui \& Hong, 2006). Mold ${ }^{2}$ descriptors have been used for the measurement of chemical space (Shen et al., 2003) and the development of QSAR models (McPhail et al., 2012; Tie et al., 2012; Ng et al., 2015). For the chemicals in ToxCast, there were 125 descriptors that had no values for the dataset and, thus, were discarded. Finally, analysis of variance (ANOVA) was used to analyze the differences among the seven modules for each of the remaining of $652 \mathrm{Mold}^{2}$ descriptors.

\subsection{Nebula}

Nebula was developed by improving the collaborative filtering recommendation algorithm ( $\mathrm{Su}$ and Khoshgoftaar 2009). In Nebula, both user-based recommendation (Wang et al., 2006) and item-based recommendation (Sarwar et al., 2001), the chemicals and assays for the ToxCast bioactivity network in this study equally contribute to the prediction. The basic concept of Nebula is that the edge values (ToxCast bioactivity data) could be inferred by their 
neighboring edges in the network. For example, the bioactivity between a chemical $c$ and an assay $a$ is predicted as $D_{p}^{c, a}$ using formula (3).

$$
D_{p}^{c a}=\frac{D_{p}^{c}+D_{p}^{a}}{2}
$$

Where $D_{p}{ }^{c}$ is the contribution that is computed using formula (4) based on the bioactivity data of other assays (except assay $a$ ) that were used to assay chemical $c$; $D_{p}{ }^{a}$ is the contribution that is calculated using formula (6) based on the bioactivity data of other chemicals (except chemical $c$ ) measured by assay $a$.

$$
D_{p}^{c}=\overline{D_{c}}+\frac{\sum_{x \in x}\left(D_{x_{a}}-\overline{D_{x}}\right) w_{c x}}{\left.\sum_{x \in X} \mid w_{c, x}\right]}
$$

In formula (4), $\overline{D_{c}}$ is the average bioactivity data of chemical $c$ measured by other assays (except $a$ ); $X$ is the set of the chemicals (except $c$ ) that have bioactivity data measured by assay $a ; D_{x, a}$ is the bioactivity data of chemical $x$ from assay $a$; $\overline{D_{x}}$ is the average bioactivity of chemical $x$ from all assays (exclude the bioactivity from assay $a$ ); $w_{c, x}$ is Pearson correlation coefficient between chemical $c$ and chemical $x$ and is calculated using formula (5).

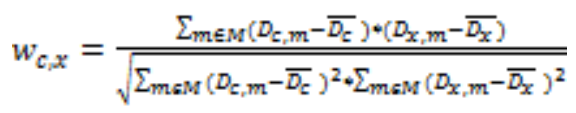

Where $M$ is the set of assays which have bioactivity data for both chemicals $c$ and $x$; $D_{c, m}$ is the bioactivity data of chemical $c$ from assay $m ; D_{x, m}$ is the bioactivity data of chemical $x$ from assay $m$.

$$
D_{p}^{a}=\overline{D_{\alpha}}+\frac{\Sigma_{y \in X}\left(D_{c, y}-\overline{D_{y}}\right] w_{y, a}}{\left.\Sigma_{y \in Y} \mid w_{y, a}\right]}
$$

In formula (6), $\overline{D_{\alpha}}$ is the average bioactivity data of all chemicals (except $c$ ) measured by assay $a ; Y$ is the set of the all assays (except $a$ ) that have bioactivity data for chemical $c ; D_{c, y}$ is the bioactivity data of chemical $c$ from assay $y ; \overline{D_{y}}$ is the average bioactivity of assay $y$ (exclude the bioactivity of chemical $c$ ); $w_{y, a}$ is Pearson correlation coefficient between assay $y$ and assay $a$ and can be computed using formula (7).

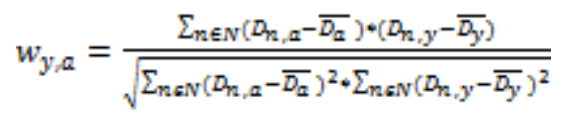

Where $N$ is the set of chemicals which have bioactivity data from both assays $a y ; D_{n, a}$ is the bioactivity data of chemical $n$ from assay $m ; D_{n, y}$ is the bioactivity data of chemical $n$ from assay $y$.

\subsection{Performance evaluation of Nebula}

We used LOO cross validation to evaluate the performance of Nebula for predicting ToxCast bioactivity data. In order to guarantee that no isolated assay or chemical existed after removing a chemical-assay datum as a testing sample, we modified the chemical-assay network. In the first step, we removed the chemicals and assays that contained only one bioactivity datum. This process was iterated until all of the chemicals and assays contained at least two bioactivity data in the final network.

In LOO cross validation, each of the ToxCast bioactivity data was taken out from the network once and only once and the remaining network was used to predict the taken-away data using Nebula. The predicted values 
were then compared with the actual ToxCast data to estimate the performance of Nebula. The prediction residual sum of squares (PRESS) was calculated using formula (8).

$$
\text { PRESS }=\sum_{i=1}^{i=n}\left(y_{i}-\hat{y}_{1}\right)^{2}
$$

Where $y$ is the actual ToxCast data, $\hat{y}$ is the predicted data, $n$ is the number of ToxCast bioactivity data. A $\mathrm{Q}^{2}$ value was then calculated using formula (9) to measure the performance of Nebula.

$$
Q^{2}=1-\frac{\text { PRESS }}{\sum_{i=1}^{i=1}\left(y-y_{i}\right)^{2}}
$$

Where $\bar{y}$ is the mean of the ToxCast bioactivity data. The average PRESS (APRESS) and the relative average PRESS (RAPRESS) were calculated using formulas (10) and (11), respectively, for application domain analysis.

$$
\begin{gathered}
\text { APRESS }=\frac{\text { PRESS }}{n} \\
\text { RAPRESS }=\frac{\text { APRESS }}{\max (y)-\min (y)}
\end{gathered}
$$

Where $\max (y)$ and $\min (\mathrm{y})$ represent the maximum and minimum values of a set of ToxCast data, respectively.

\subsection{Application domain analysis}

According to the assay description file of ToxCast, the assays could be categorized into different groups according to assay description terms (tissue, biological process target, assay target family). Nebula could have different performance for the bioactivity data of the assays with different description terms. Identifying the assay description terms with higher prediction performance of Nebula is important for better utilization of the algorithm. Therefore, we calculated the APRESS and RAPRESS for the data with different assay description terms.

\section{Results}

\subsection{Modularity analysis of ToxCast bioactivity data network}

We constructed a bipartite network using the ToxCast bioactivity data $\left(-\log \mathrm{AC}_{50}, \mathrm{AC}_{50}<1,000,000\right)$. In this bipartite network, chemicals and assays were presented as nodes and the bioactivity data were treated as edges linking the chemicals and assays. The network had 32,763 bioactivity data between 1,799 chemicals and 544 assays. Modularity analysis was used to decompose the network into seven densely connected modules as shown in Figure 2 with a modularity value of 0.3152 . The chemicals, assays and bioactivity data in the seven modules are summarized in Table 1 and the module assignment for the 544 assays and the 1,799 chemicals are given in supplementary Table S1 and S2, respectively.

\subsection{Permutation tests}

In the first type of permutation tests, the 32,763 ToxCast bioactivity data were randomly assigned to the 1799 chemicals and 544 assays, yielding a random network with different topological properties. A modularity value was calculated for the random network for comparison with the modularity value obtained from the real ToxCast bioactivity data network. To make the evaluation statistically robust, 10,000 such random networks were generated for modularity analysis. The modularity values yielded from the 10,000 random networks are given in Figure 3A, with a mean of 0.1705 and standard deviation of 0.0021 .

The second type of permutation tests used random networks with the same topological properties of the real ToxCast bioactivity data network. The degree distribution of chemicals and assays were kept the same while the 
32,763 ToxCast bioactivity data were randomly shuffled to generate a random network for modularity analysis. To make the modularity value statistically representative, 10,000 such random networks were generated and their modularity values were calculated. Figure 3B shows the distribution of the 10,000 modularity values with a mean of 0.1325 and a standard deviation of 0.0018 .

Comparing the modularity value of ToxCast bioactivity data network with the modularity values from the random networks indicated that ToxCast bioactivity data network had a significantly larger modularity value than the random networks with Z-scores of 68.90, 101.50 for comparing to the first type and the second type of random networks, respectively, revealing that the seven modules decomposed from ToxCast bioactivity data network could not be obtained by chance and thus the network contained useful information characterizing chemicals and assays in the modules.

\subsection{Assay enrichment in modules}

The enriched assay description terms in the seven modules are given in Figure 4A. Generally, module 1 and module 2 had relatively more enriched terms.

There are seven terms in module 1, including two assay target families, two biological process targets and three tissues. Ovary, cervix and kidney were the three tissues most frequently used in the assays in module 1 . In addition, all of the three ESR1/ERE binding assays were enriched in module 1.

Module 2 contained ten different assay terms, including five assay target families, four biological process targets and one tissue. It is worth noting that all of the oxidase and cell death related assays are contained in this module. GPCR was the largest target family in module 2 with more than $90.67 \%$ covered in this module. Brain is the only tissue contained in module 2 .

Module 3 contained three assay terms. The assays were mainly focused on regulating the cell nucleus process, including regulation of gene expression and transcription factor.

Module 4 enriched three assay terms. The largest components of transcription factors in the network were included in module 4. More interestingly, of the 93 assays in this module, the tissue of 82 is liver and the assay target family of 80 is transcription factors and their biological process target is regulation of transcription factor activity.

Assay term cytochrome p450 was exclusively enriched in module 5. Specifically, $87.88 \%$ of assays targeting CYP450 in the network were included in module 5.

Module 6 showed a preference for regulating catalytic activity of kinase and phosphatase. Notably, $93.75 \%$ assays in kinase term was contained in this module.

Module 7 contained only eight assays which were all targeted nuclear receptors.

\subsection{Pathway enrichment in modules}

The pathway enrichment analysis by DAVID identified the enriched AOPs in the modules displayed in Figure 4B. The endocrine system pathways, metabolic pathways and bile secretion pathways were enriched in modules 1,5 and 7 , respectively. The estrogen signaling pathway was also enriched in module 1 . The majority of the pathways contained in Module 5 were of the three types of metabolic pathways: lipid metabolism, amino acid metabolism and xenobiotics metabolism. Module 2 included four synapse related pathways in the brain. Module 6 had the most pathways (56 pathways) which were mainly on the immune system, cellular process, signal transduction, endocrine system and nervous system. Module 3 and module 4 contained fewer pathways than other modules. Circadian 
rhythm pathway was the only one included in module 3. The signaling transduction pathways, namely, Wnt signaling pathway, TGF-beta signaling pathway and Hippo signaling pathway were included in module 4.

\subsection{Chemical structure difference in modules}

To examine the chemical structure difference between the seven modules, the $777 \mathrm{Mold}^{2}$ molecular descriptors were calculated for each of the 1799 chemicals. After discarding 125 descriptors (such as number of 9-membered rings) that do not have values for the 1799 chemicals, 652 descriptors were used in ANOVA. The p-values from ANONA were used to rank the 652 descriptors to find the significant structural features that drive the decomposition of the network into the seven modules. The ranked p-values of the $652 \mathrm{Mold}^{2}$ molecular descriptors are listed in supplementary Table S3. Of the 652 descriptors, 548 (84\%) had a p-value less than 0.05 and 454 (70\%) had a Bonferroni corrected p-value (the most conservative correction for multiple tests) less than 0.05 , indicating the chemical structures are different between the seven modules. As an example, the distributions of D595 and D358 (the top two descriptors) are plotted in Figure 5A and 5B, respectively. Chemicals in module 7 have a large volume (represented by the descriptor of molecular multiple path count) and contain many atoms with high polarizability, while chemicals in module 3 contain less atoms with high polarizability and chemicals in module 1 are small in size. Chemicals in modules 5 and 6 are relatively smaller than the chemicals in Module 7 and have more atoms with high polarizability than chemicals in modules 1 to 4 but less than model 7. Chemicals in module 2 are medium in both molecular size and polarizability. Chemicals in model 4 are similar to the chemicals in modules 5 and 6 in size but have lower polarizability.

\subsection{Performance of Nebula}

To evaluate the performance of Nebula for prediction of ToxCast bioactivity data, we conducted LOO cross validations. To ensure the network is not disconnected after removing one ToxCast datum as a testing sample, every chemical and assay must have at least two data. Applying this criterion yielded a network of 32,615 data that connect 1,699 chemicals and 496 assays for the LOO cross validation. The Nebula predicted values from the LOO cross validation are plotted on the y-axis against the actual ToxCast data (x-axis) in Figure 6. For most of the 32,615 ToxCast data, Nebula predicted the data well and yielded a good $\mathrm{Q}^{2}$ of 0.5416 and a Pearson correlation coefficient of 0.7406 between the actual ToxCast data and the Nebula predictions, indicating ToxCast bioactivity data could be predicted by Nebula and, thus, predictive models based on ToxCast data could be applied to chemicals that have not been tested in ToxCast project.

To test the performance difference of Nebula among the seven modules yielded from the modularity analysis, we extracted the ToxCast data within each of the seven modules and then compared the actual ToxCast data with the Nebula predictions as plotted in Figure 7. The Nebula prediction performance metrics, $Q^{2}$ values and the Pearson correlation coefficients for the seven modules are given in Table 1. It was observed that Nebula performed much worse in the modules of much smaller size (modules 7 and 6), indicating lack of enough similar data for training reduced Nebula prediction performance. For the modules with a large amount of data, Nebula performed well.

The pathway enrichment analysis revealed that certain pathways were enriched in different modules. To test Nebula performance in different pathways, we partitioned ToxCast data into the 24 main pathways. The actual ToxCast data and the corresponding Nebula predictions in each of the 24 main pathways were plotted in the supplementary Figure S1-S24. The number of assays, number of data, and the Nebula prediction performance metrics $\left(Q^{2}\right.$ values and the Pearson correlation coefficients) for the 24 main pathways are given in Table 2 . It is a bit surprising that the number of ToxCast assays or data in a pathway did not impact Nebula performance much, which was observed in different modules. The biological characteristics of a pathway showed importance for Nebula performance. The pathways "Cell growth and death", "Folding, sorting and degradation" and "Endocrine system" 
showed good Nebula performance, while the pathway "Xenobiotics biodegradation and metabolism" had a low performance.

\subsection{Application domain analysis}

The LOO cross validations (Figure 6) indicated that some predictions were better than the others. To find what data could be better predicted by Nebula, application domain analysis was conducted on the LOO cross validations. We analyzed the prediction performance on the data with different assay description terms. The ToxCast bioactivity data were first separated into different groups according to their assay target family, biological process target and tissue, respectively. APRESS and RAPRESS were then calculated for each of the groups and the results were summarized in supplementary Tables S4, S5 and S6 for the groups based on assay target family, biological process target and tissue, respectively.

Figure 7A shows APRESS and RAPRESS for target families used in the ToxCast assays. Of the 31 target families used in the assays, $21(67.7 \%)$ had a smaller RAPRESS than the average RAPRESS, indicating ToxCast data from assays utilizing the target families could be better predicted by Nebula. Especially, the data from the assay "NVS_ENZ_rACFSKBinding" that included the description term lyase could be predicted very well with an average prediction error (in PRESS) of about $1 \%$ of the data range. Notably, 11,921 bioactivity data were generated from 122 assays that utilized transcription factors and these were predicted well by Nebula with an average prediction error of about $2.5 \%$ of the data range.

The ToxCast bioactivity data were from assays involving 12 biological processes which had different prediction performance as depicted in Figure 7B. For the data from assays of two thirds of the biological processes, Nebula showed higher prediction performance than its overall prediction performance. The 122 assays involving regulation of transcription factor activity generated 11,694 bioactivity data that were best predicted by Nebula with an average prediction error (in PRESS) of about $2.6 \%$ of the data range.

Nebula performed differently for the ToxCast bioactivity data obtained from assays using different tissues as shown in Figure 7C. Off the 18 tissues used in ToxCast, the data from assays of 8 tissues (44.4\%) had better predictions than the overall performance. Especially, the 15,958 bioactivity data from assays using liver were well predicted by Nebula with an average prediction error (in PRESS) of about $3.6 \%$ of the data range.

To ensure the performance difference was from assay types rather from the number of data, we examined correlations between RAPRESS and number of data. The Pearson correlation coefficient values were 0.2149, 0.1977 and -0.0766 for target family, biological process and tissue (assay description terms), respectively, indicating that number of the data does not impact prediction performance of Nebula. Assays were the cause of the difference in Nebula performance.

\section{Discussion}

Modules detected by topological structures have demonstrated the ability to facilitate understanding biological networks (Barabasi and Oltvai 2004; Newman 2012). The hypothesis behind this is that a topological module corresponds to a function module (Barabási et al., 2011). We applied modularity analysis to the ToxCast bioactivity data network and identified seven distinct modules. Those seven modules help our understanding of the assays and chemicals in ToxCast because the assays and chemicals should be similar in the same modules and distinct between the modules in terms of their bioactivity data.

Permutation testing is a powerful statistical approach to estimate chance correlation among the data used in analysis and is widely used in supervised machine learning studies (Hong et al. 2004). Compared to the permutation tests on the modularity of random networks, the significantly larger modularity of the chemical-assay network in ToxCast indicates the topological structure was formed by the chemical-assay bioactivities in ToxCast. The ToxCast 
bioactivity data network is unlikely formed by chance and instead contains signal for the relationship between the assays and the chemicals. Therefore, ToxCast data should be very useful in understanding toxicological profiles of environmental chemicals.

Exploring the network topological structure may provide some insights into the chemical-assay bioactivity. Similar to the published protein-protein interaction networks (Chen and Yuan 2006; Yook et al., 2004) and metabolic networks (Guimera and Amaral 2005; Ma et al., 2004), the seven topological modules detected in the bipartite network of ToxCast bioactivity data were found to display specific properties of the chemicals and assays. For example, metabolism pathways (especially drug metabolism through CYP450) were exclusively enriched in module 5. The majority of the assays in module 4 came from liver tissue. Our pathway enrichment study suggested that the seven modules could help facilitate AOPs studies of specific toxicity endpoints such as liver related toxicity or drug metabolism related disorder.

Inspired by the relationship between network topology and biological information discovered in the modularity analysis, Nebula was developed to predict chemical-assay bioactivity based on network topological information. The basic hypothesis is that chemical-assay bioactivity data can be inferred by the bioactivities of neighboring chemicals and assays. The overall $\mathrm{Q}^{2}=0.5416$ achieved in the LOO cross validations suggested that Nebula showed certain power on predicting chemical-assay bioactivity.

Application domain analysis indicated that Nebula displayed preference on the bioactivity data of several assays, such as the assays on the regulation of transcription factor activity, the assays using liver tissue and the assays targeting lyase. Furthermore, the performance difference among assays was not impacted by the data volume. For example, assays with description term lyase had only 11 bioactivity data while the assays utilizing transcription factor had much more data (11,921 bioactivity data). Nebula achieved good predictive performance for both types of data.

\section{Conclusion}

We identified seven modules in the chemical-assay network constructed from ToxCast data. The modules showed distinct properties for assays and chemicals. We showed that network analysis is a promising approach to understanding ToxCast data. We developed Nebula based on using topological linkage for predicting ToxCast bioactivity data. The good prediction performance suggested that Nebula was applicable and suitable for predicting ToxCast data. Application domain analysis revealed that Nebula could predict ToxCast data well from specific assays. Our results demonstrated Nebula could infer bioactivity data for chemicals not contained in ToxCast, allowing better utilization of ToxCast data in the risk assessment of environmental chemicals.

\section{Acknowledgement}

This research was supported in part by an appointment to the Research Participation Program at the National Center for Toxicological Research (Hao Ye, Heng Luo and Hui Wen Ng) administered by the Oak Ridge Institute for Science and Education through an interagency agreement between the U.S. Department of Energy and the U.S. Food and Drug Administration. The content is solely the responsibility of the authors and does not necessarily represent the official views of the Food and Drug Administration.

\section{Reference:}

Abdelaziz, A., Sushko, Y., Novotarskyi, S., Körner, R., Brandmaier, S., Tetko, I., 2015. Using Online Tool (iPrior) for Modeling ToxCast ${ }^{\mathrm{TM}}$ Assays Towards Prioritization of Animal Toxicity Testing. Comb. Chem. High Throughput Screen. 18, 420-438.

ATSDR U.S., 2014. Chemicals, Cancer, and You. 1-12. 
Barabási, A.-L., Gulbahce, N., Loscalzo, J., 2011. Network medicine: a network-based approach to human disease. Nat. Rev. Genet. 12, 56-68.

Barabasi, A.-L, Oltvai, Z.N., 2004. Network biology: understanding the cell's functional organization. Nat. Rev. Genet. 5, 101-113.

Berg, E.L., Yang, J., Polokoff, M.A., 2013. Building predictive models for mechanism-of-action classification from phenotypic assay data sets. J. Biomol. Screen. 18, 1260-1269.

Blondel, V.D., Guillaume, J.-L., Lambiotte, R., Lefebvre, E., 2008. Fast unfolding of communities in large networks. J. Stat. Mech., P10008.

Carson, R., 1962. Silent Spring. Houghton Mifflin, September 27..

CDC, 2015. U.S. Fourth National Report on Human Exposure to Environmental Chemicals, Updated Tables, February..

Chen, J., Yuan, B., 2006. Detecting functional modules in the yeast protein-protein interaction network. Bioinformatics. 22, 2283-2290.

Dix, D.J., Houck, K.A., Martin, M.T., Richard, A.M., Setzer, R.W., Kavlock, R.J., 2007. The ToxCast program for prioritizing toxicity testing of environmental chemicals. Toxicol. Sci. 95, 5-12.

Guimera, R., Amaral, L.A.N., 2005. Functional cartography of complex metabolic networks. Nature. 433, 895-900.

Hong, H., Xin, X., 1990. ESSESA: an expert system for structure elucidation from spectra analysis. 1. The knowledge base of infrared spectra and analysis and interpretation program. J. Chem. Inf. Comput. Sci. 30, 203-210.

Hong, H., Xin, X., 1992a. ESSESA: an expert system for structure elucidation from spectra analysis. 2. A novel algorithm of perception of the linear independent smallest set of smallest rings. Analytica. Chimica. Acta. 262, 179-191.

Hong, H., Xin, X., 1992b. ESSESA: an expert system for structure elucidation from spectra analysis. 3. LNSCS for chemical knowledge representation. J. Chem. Inf. Comput. Sci. 32, 116-120.

Hong, H., Xin, X., 1994a. ESSESA: an expert system for structure elucidation from spectra analysis. 4. Canonical representation of structures. J. Chem. Inf. Comput. Sci. 34, 730-734.

Hong, H., Xin, X., 1994b. ESSESA: an expert system for structure elucidation from spectra analysis. 5. Substructure constraints from from analysis of first-order $1 \mathrm{H}-\mathrm{NMR}$ spectra. J. Chem. Inf. Comput. Sci. 34, 1259-1266.

Hong, H., Han, Y., Xin, X., Shi, Y., 1995. ESSESA: an expert system for structure elucidation from spectra. 6. Substructure constraints from analysis of 13C-NMR spectra. J. Chem. Inf. Comput. Sci. 35, 9791000.

Hong, H., Xie, Q., Ge, W., Qian, F., Fang, H., Shi, L., Su, Z., Perkins, R., Tong, W., 2008. Mold2, molecular descriptors from 2D structures for chemoinformatics and toxicoinformatics. J. Chem. Inf. Model. 48, 1337-1344.

Huang da, W., Sherman, B.T., Lempicki, R.A., 2009. Systematic and integrative analysis of large gene lists using DAVID bioinformatics resources. Nat. Protoc. 4, 44-57.

Kanehisa, M., Goto, S., Sato, Y., Furumichi, M., Tanabe, M., 2011. KEGG for integration and interpretation of large-scale molecular data sets. Nucleic Acids Res. D109-D114.

Kavlock, R., Chandler, K., Houck, K., Hunter, S., Judson, R., Kleinstreuer, N., Knudsen, T., Martin, M., Padilla, S., Reif, D., 2012. Update on EPA's ToxCast program: providing high throughput decision support tools for chemical risk management. Chem. Res. Toxicol. 25, 1287-1302.

Kleinstreuer, N., Smith, A., West, P., Conard, K., Fontaine, B., Weir-Hauptman, A., Palmer, J., Knudsen, T., Dix, D., Donley, E., 2011. Identifying developmental toxicity pathways for a subset of ToxCast chemicals using human embryonic stem cells and metabolomics. Toxicol. Appl. Pharmacol. 257, 111-121. 
Kleinstreuer, N.C., Dix, D.J., Houck, K.A., Kavlock, R.J., Knudsen, T.B., Martin, M.T., Paul, K.B., Reif, D.M., Crofton, K.M., Hamilton, K., 2012. In vitro perturbations of targets in cancer hallmark processes predict rodent chemical carcinogenesis. Toxicol. Sci. 131, 40-55.

Kleinstreuer, N.C., Yang, J., Berg, E.L., Knudsen, T.B., Richard, A.M., Martin, M.T., Reif, D.M., Judson, R.S., Polokoff, M., Dix, D.J., 2014. Phenotypic screening of the ToxCast chemical library to classify toxic and therapeutic mechanisms. Nat. Biotechnol. 32, 583-591.

Liu, J., Mansouri, K., Judson, R.S., Martin, M.T., Hong, H., Chen, M., Xu, X., Thomas, R., Shah, I., 2015. Predicting hepatotoxicity using ToxCast in vitro bioactivity and chemical structure. Chem. Res. Toxicol. 20, 738-751.

Ma, H.-W., Zhao, X.-M., Yuan, Y.-J., Zeng, A.-P., 2004. Decomposition of metabolic network into functional modules based on the global connectivity structure of reaction graph. Bioinformatics. 20, 1870-1876.

Martin, M.T., Knudsen, T.B., Reif, D.M., Houck, K.A., Judson, R.S., Kavlock, R.J., Dix, D.J., 2011. Predictive model of rat reproductive toxicity from ToxCast high throughput screening. Biol. Reprod. 85, 327339.

Masui, H., Hong, H., 2006. Spec2D: a structure elucidation system based on $1 \mathrm{H}$ NMR and H-H COSY spectra in organic chemistry. J. Chem. Inf. Model. 46, 775-787.

McPhail, B., Tie, Y., Hong, H., Pearce, B.A., Schnackenberg, L.K., Ge, W., Valerio, L.G., Fuscoe, J.C., Tong, W., Buzatu, D.A., et al., 2012. Modeling chemical interaction profiles: I. Spectral data-activity relationship and structure-activity relationship models for inhibitors and non-inhibitors of cytochrome P450 CYP3A4 and CYP2D6 isozymes. Molecules. 17, 3283-3406.

Muir, D.C., Howard, P.H., 2006. Are there other persistent organic pollutants? A challenge for environmental chemists. Environ. Sci. Technol. 40, 7157-7166.

Newman, M., 2012. Communities, modules and large-scale structure in networks. Nat. Phys. 8, 25-31.

Ng, H.W., Doughty, S.W., Luo, H., Ye, H., Ge, W., Tong, W., Hong, H., 2015. Development and Validation of Decision Forest Model for Estrogen Receptor Binding Prediction of Chemicals Using Large Data Sets. Chem. Res. Toxicol. 28, 2343-2351.

Sarwar, B., Karypis, G., Konstan, J., Riedl, J., 2001. Item-based collaborative filtering recommendation algorithms. Proceedings of the 10th international conference on World Wide Web. ACM New York. 501-508.

Shah, F., Greene, N., 2013. Analysis of Pfizer compounds in EPA's ToxCast chemicals-assay space. Chem. Res. Toxicol. 27, 86-98.

Shen, J.,Xu, L., Fang, H., Richard, A.M., Bray, J.D., Judson, R.S., Zhou, G., Colatsky, T.J., Aungst, J.L., Teng, C., Harris, S.C., Ge, W., Dai, S.Y., Su, Z., Jacobs, A.C., Harrouk, W., Perkins, R., Tong, W., Hong, H., 2013. EADB: An Estrogenic Activity Database for Assessing Potential Endocrine Activity. Toxicol. Sci. 135, 277-291.

Sipes, N.S., Martin, M.T., Reif, D.M., Kleinstreuer, N.C., Judson, R.S., Singh, A.V., Chandler, K.J., Dix, D.J., Kavlock, R.J., Knudsen, T.B., 2011. Predictive models of prenatal developmental toxicity from ToxCast high-throughput screening data. Toxicol. Sci. 124, 109-127..

Su, X., Khoshgoftaar, T.M., 2009. A survey of collaborative filtering techniques. Adv. Art. Int. 2009, Article ID 421425.

Tie, Y., McPhail, B., Hong, H., Pearce, B.A., Schnackenberg, L.K., Ge, W., Buzatu, D.A., Wilkes, J.G., Fuscoe, J.C., Tong, W., et al., 2012. Modeling chemical interaction profiles: II. Molecular docking, spectral data-activity relationship, and structure-activity relationship models for potent and weak inhibitors of cytochrome p450 cyp3A4 isozyme. Molecules. 17, 3407-3460.

TSCA., 2015. TSCA Chemical Substance Inventory. (http://www.epa.gov/tsca-inventory/about-tscachemical-substance-inventory) 
Wang, J., De Vries, A.P., Reinders, M.J., 2006. Unifying user-based and item-based collaborative filtering approaches by similarity fusion. Proceedings of the 29th annual international ACM SIGIR conference on Research and development in information retrieval. ACM New York. 501-508.

Yook, S.H., Oltvai, Z.N., Barabási, A.L., 2004. Functional and topological characterization of protein interaction networks. Proteomics. 4, 928-942.

Zang, Q., Rotroff, D.M., Judson, R.S., 2013. Binary classification of a large collection of environmental chemicals from estrogen receptor assays by quantitative structure-activity relationship and machine learning methods. J. Chem. Inf. Model. 53, 3244-3261. 
Table 1 Summary of the seven modules

\begin{tabular}{|l|l|l|l|l|l|}
\hline \multirow{2}{*}{ Module } & \multirow{2}{*}{ Chemicals } & \multirow{2}{*}{ Assays } & \multirow{2}{*}{ Data } & \multicolumn{2}{|l|}{ Nebula performance } \\
\cline { 5 - 6 } & & & & $\mathrm{Q}^{2}$ & $\mathrm{R}^{*}$ \\
\hline Module 1 & 422 & 53 & 3323 & 0.6274 & 0.8023 \\
\hline Module 2 & 243 & 192 & 5960 & 0.4735 & 0.6895 \\
\hline Module 3 & 461 & 43 & 1641 & 0.5594 & 0.7501 \\
\hline Module 4 & 458 & 93 & 3993 & 0.6637 & 0.8218 \\
\hline Module 5 & 173 & 60 & 1729 & 0.6126 & 0.7923 \\
\hline Module 6 & 35 & 95 & 484 & 0.3103 & 0.5582 \\
\hline Module 7 & 7 & 8 & 45 & -0.2293 & 0.0835 \\
\hline
\end{tabular}

*Pearson correlation coefficient between the actual data and the predicted values 
Table 2 Nebula performance in the main pathways

\begin{tabular}{|l|l|l|l|l|}
\hline \multirow{2}{*}{ Pathway } & \multirow{2}{*}{ Assays } & \multirow{2}{*}{ Data } & \multicolumn{2}{l}{ Nebula performance } \\
\cline { 5 - 6 } & & & Q2 & R \\
\hline Cell growth and death & 37 & 1008 & 0.6272 & 0.7955 \\
\hline Folding, sorting and degradation & 5 & 1283 & 0.6242 & 0.8003 \\
\hline Endocrine system & 120 & 8958 & 0.6073 & 0.7842 \\
\hline Excretory system & 31 & 4472 & 0.5903 & 0.7745 \\
\hline Development & 37 & 1570 & 0.5516 & 0.7439 \\
\hline Nervous system & 65 & 2467 & 0.5342 & 0.7323 \\
\hline Environmental adaptation & 11 & 288 & 0.5322 & 0.736 \\
\hline Signal transduction & 140 & 5034 & 0.5293 & 0.731 \\
\hline Cellular community & 64 & 1292 & 0.5215 & 0.7243 \\
\hline Immune system & 47 & 780 & 0.5064 & 0.712 \\
\hline Lipid metabolism & 16 & 1235 & 0.4832 & 0.6969 \\
\hline Transport and catabolism & 23 & 351 & 0.4558 & 0.6765 \\
\hline Digestive system & 19 & 434 & 0.4107 & 0.6413 \\
\hline Signaling molecules and interaction & 59 & 2258 & 0.3468 & 0.5906 \\
\hline Sensory system & 12 & 215 & 0.3261 & 0.5735 \\
\hline Circulatory system & 19 & 289 & 0.2763 & 0.5266 \\
\hline Cell motility & 14 & 352 & 0.2342 & 0.4934 \\
\hline Metabolism of cofactors and vitamins & 11 & 752 & 0.1282 & 0.3865 \\
\hline Carbohydrate metabolism & 2 & 36 & 0.1257 & 0.4083 \\
\hline Xenobiotics biodegradation and metabolism & 13 & 805 & 0.0922 & 0.3529 \\
\hline Amino acid metabolism & 3 & 126 & 0.0682 & 0.3932 \\
\hline Biosynthesis of other secondary metabolites & 2 & 97 & -0.0384 & 0.3112 \\
\hline Nucleotide metabolism & 3 & 73 & -0.1696 & 0.0171 \\
\hline Translation & 2 & 11 & -0.374 & 0.3833 \\
\hline Pearson corm & 23 \\
\hline
\end{tabular}

*Pearson correlation coefficient between the actual data and the predicted values 


\section{Figure legend}

Figure 1. Study design. The chemical-assay network was constructed based on the quantitative chemical-assay bioactivity data in ToxCast. Modularity analysis was then conducted to identify modules with distinct topological properties. Permutation testing was conducted using 10,000 randomly generated networks to assess chance correlation. Thereafter, the assays and chemicals in the modules were examined using enrichment analysis. Finally, Nebula was developed and applied to predict the bioactivity data in ToxCast.

Figure 2. ToxCast bioactivity data network and modularity analysis results were plotted using Cytoscape 3.2.0. The network was displayed on the left and the yielded modules were given on the right. The nodes represent chemicals and assays. The edges indicate the bioactivity data. The chemicals and assays in the seven modules were marked in different colors.

Figure 3. Modularity distributions of 10,000 permutations for random networks in which the degrees of the nodes were not fixed (A) and the random networks with the same degrees of nodes of the ToxCast bioactivity data network (B). The graphs were plotted using python package matplotlib.

Figure 4. Enrichment analysis of assays in the seven modules. (A) Modules and the enriched assay description terms. The blue rectangles represent modules. The green, red and purple ellipses indict the assay description terms of assay_target_family, biological_proces_target and tissue, respectively. (B) Modules and the enriched pathways. The blue rectangles represent modules. Pathways were represented by ellipses and marked in different colors according the twelve categories in KEGG.

Figure 5. Distributions of molecular descriptors in the seven modules (A) for descriptor D595 (highest eigenvalue of Burden matrix weighted by atomic polarizabilities) and (B) for descript D358 ( molecular multiple path count of order 10).

Figure 6. LOO cross validation results of Nebula. The points represent the chemical-assay pairs having bioactivity data in ToxCast. The $\mathrm{x}$ axis gave the actual bioactivity data and the corresponding predicted values from Nebula were indicated by the $y$ axis.

Figure 7. Nebula performance in different modules. The actual $-\log (\mathrm{AC} 50)$ values from ToxCast (x-axis) were plotted against the Nebula predicted $-\log (\mathrm{AC} 50)$ values (y-axis) for modules 1-6 indicated by the labels in the upleft corner in each panel. Model 7 was not shown as it contained very few data.

Figure 8. Application domain analysis results. The APRESS (circles) and RAPRESS (up-triangles) depicted at the yaxis were plotted against the groups of assays given at the $\mathrm{x}$-axis for assay description terms according to assay target family terms (A), biological_process_target terms (B) and tissue (C). 

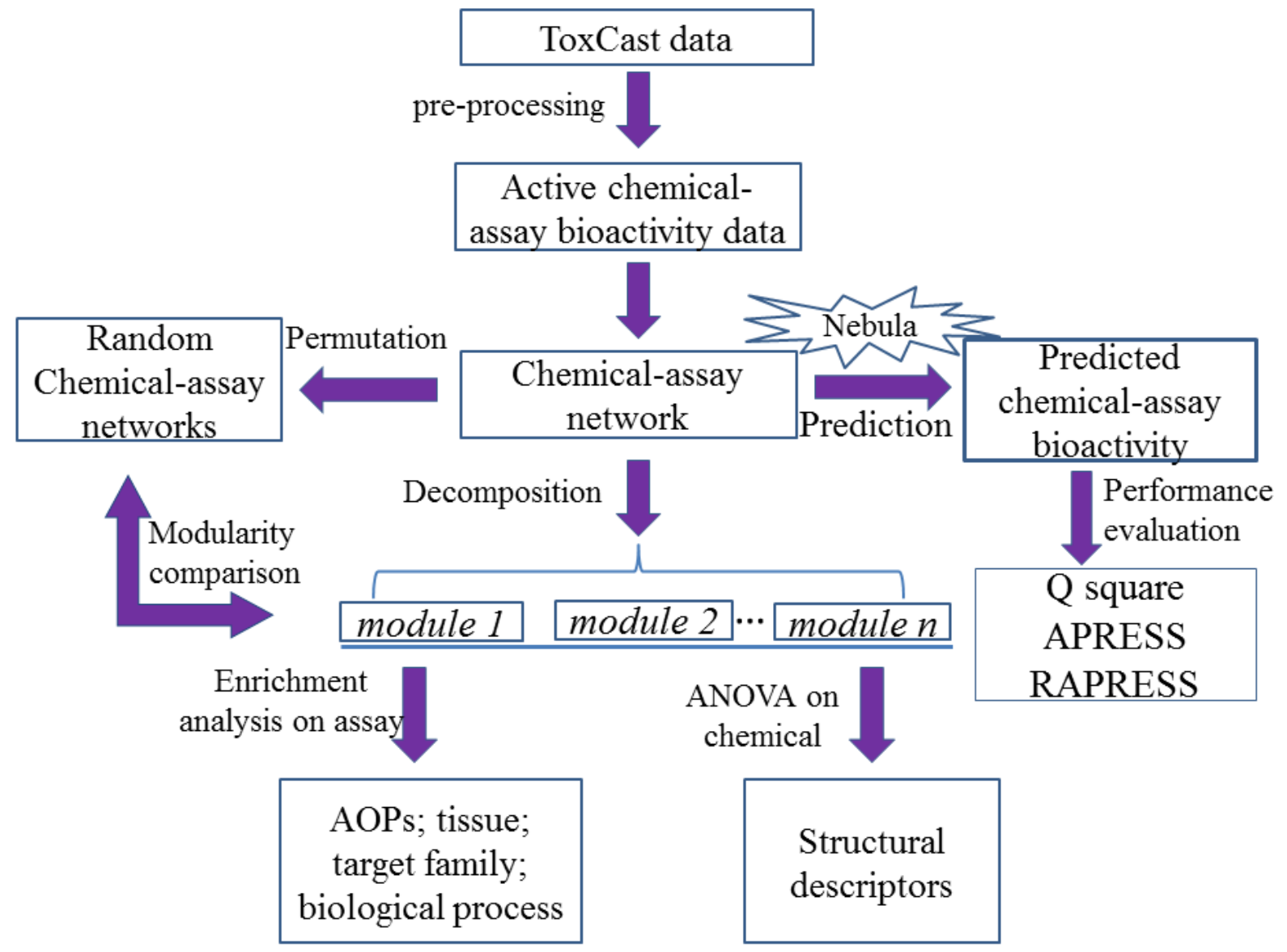

Figure 1 


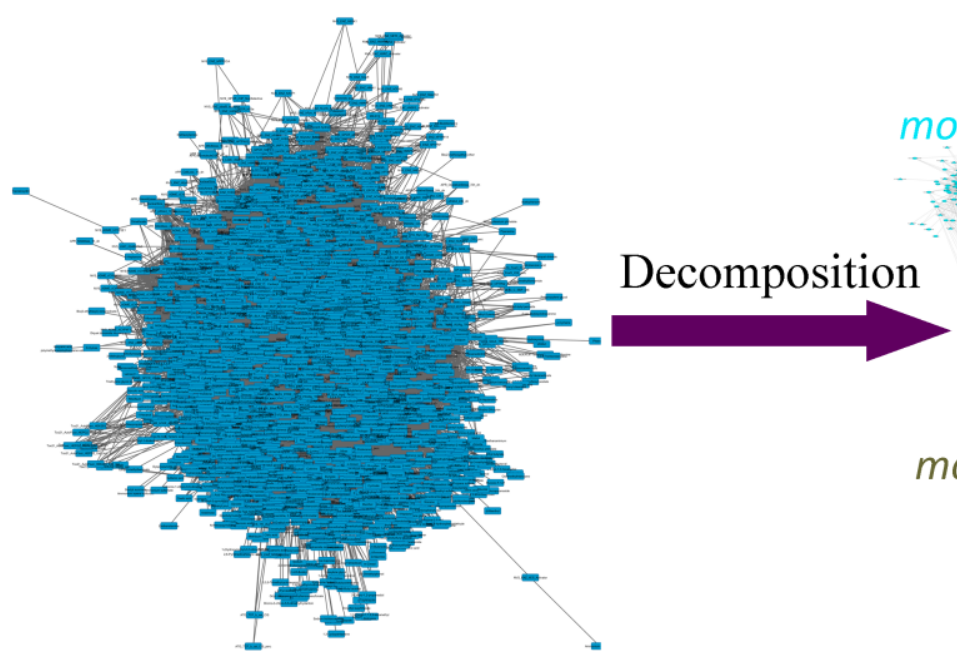

module 1

module 5

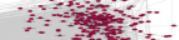

into

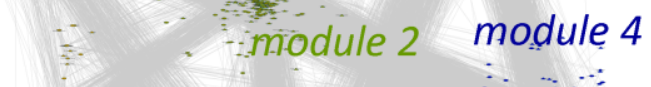

module 7

module 6

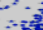

㰵

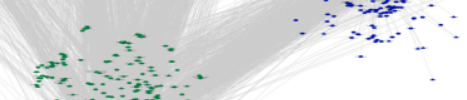

nis.

module 3

Figure 2 

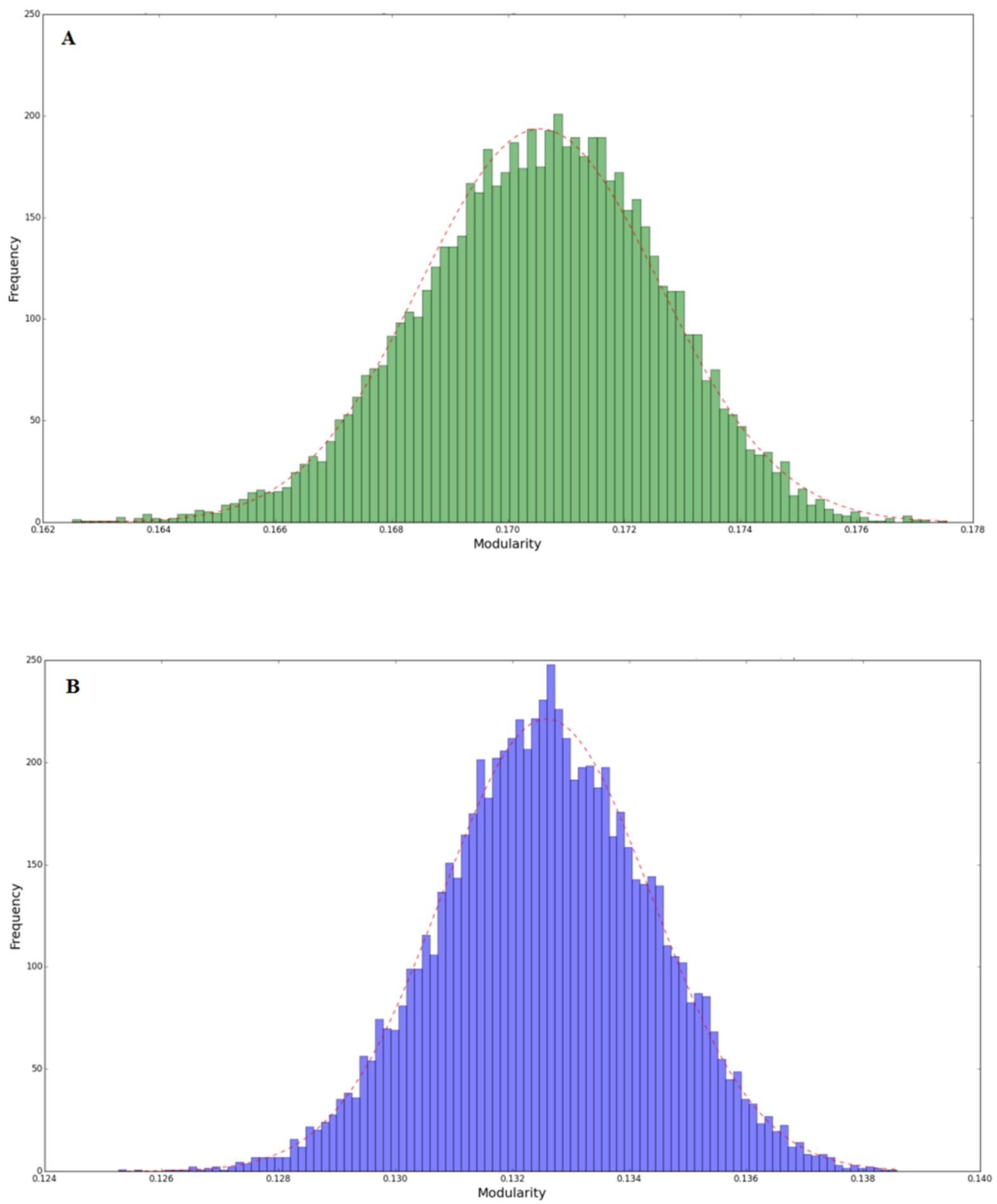

Figure 3 

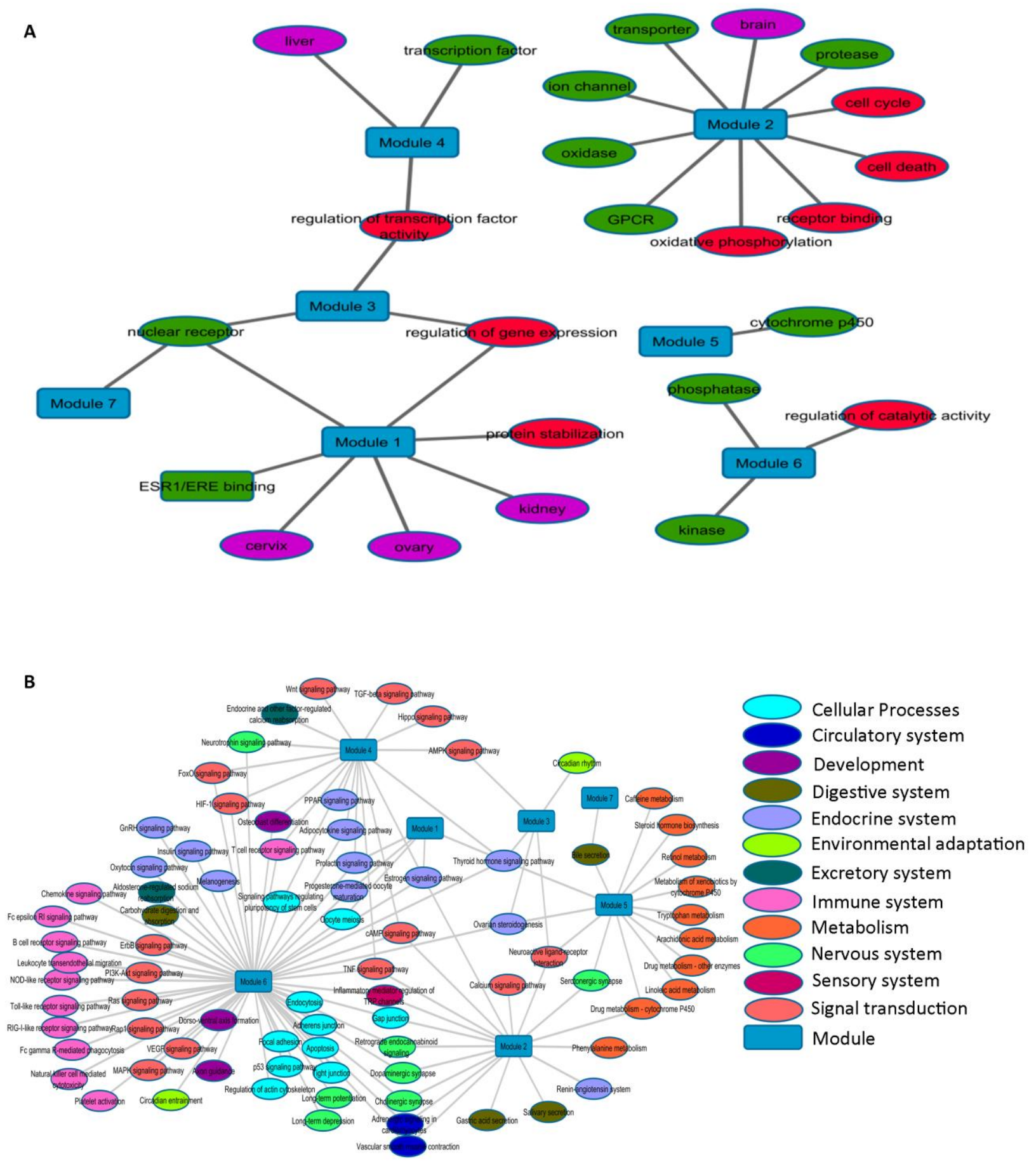

Figure 4 

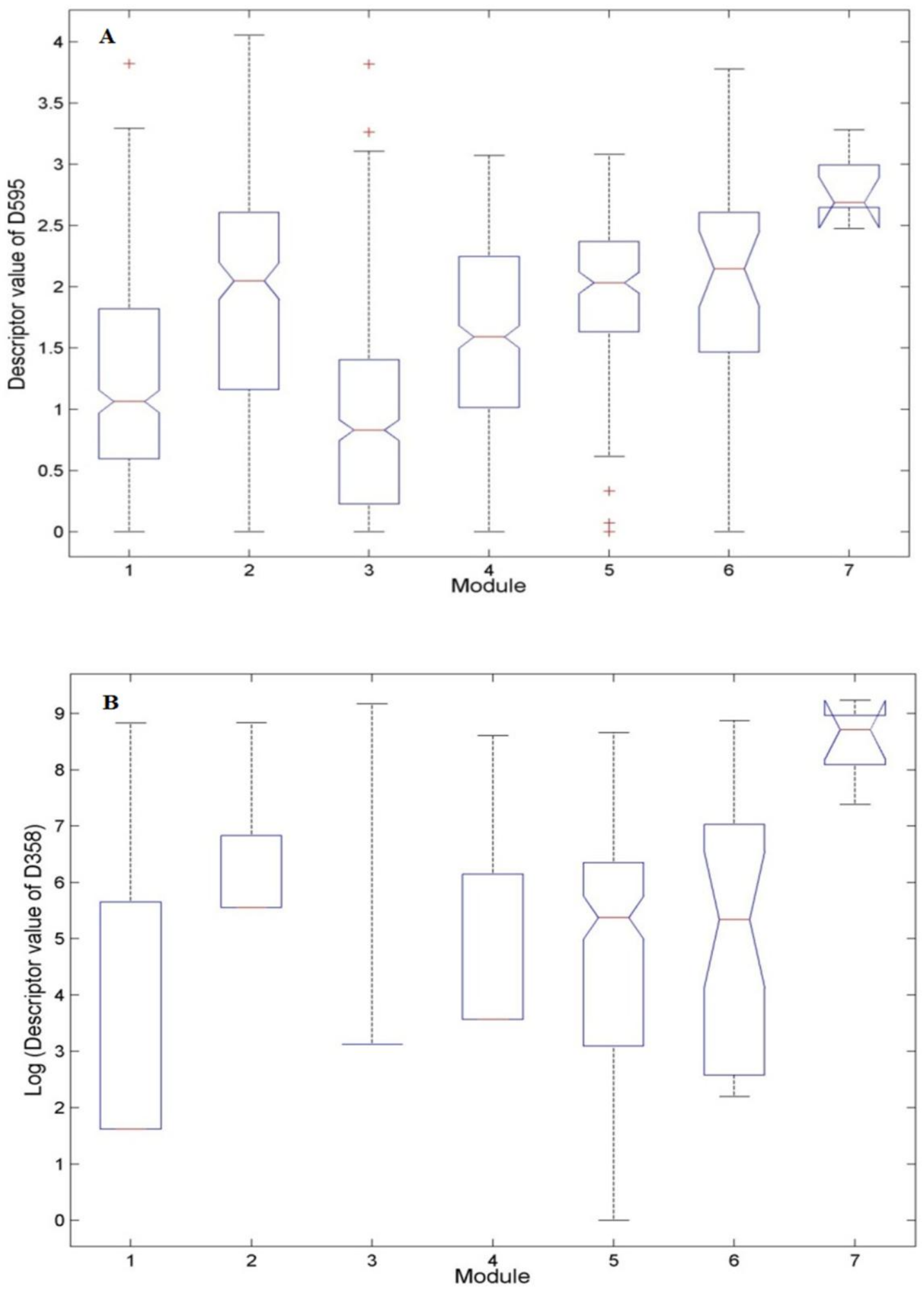

Figure 5 


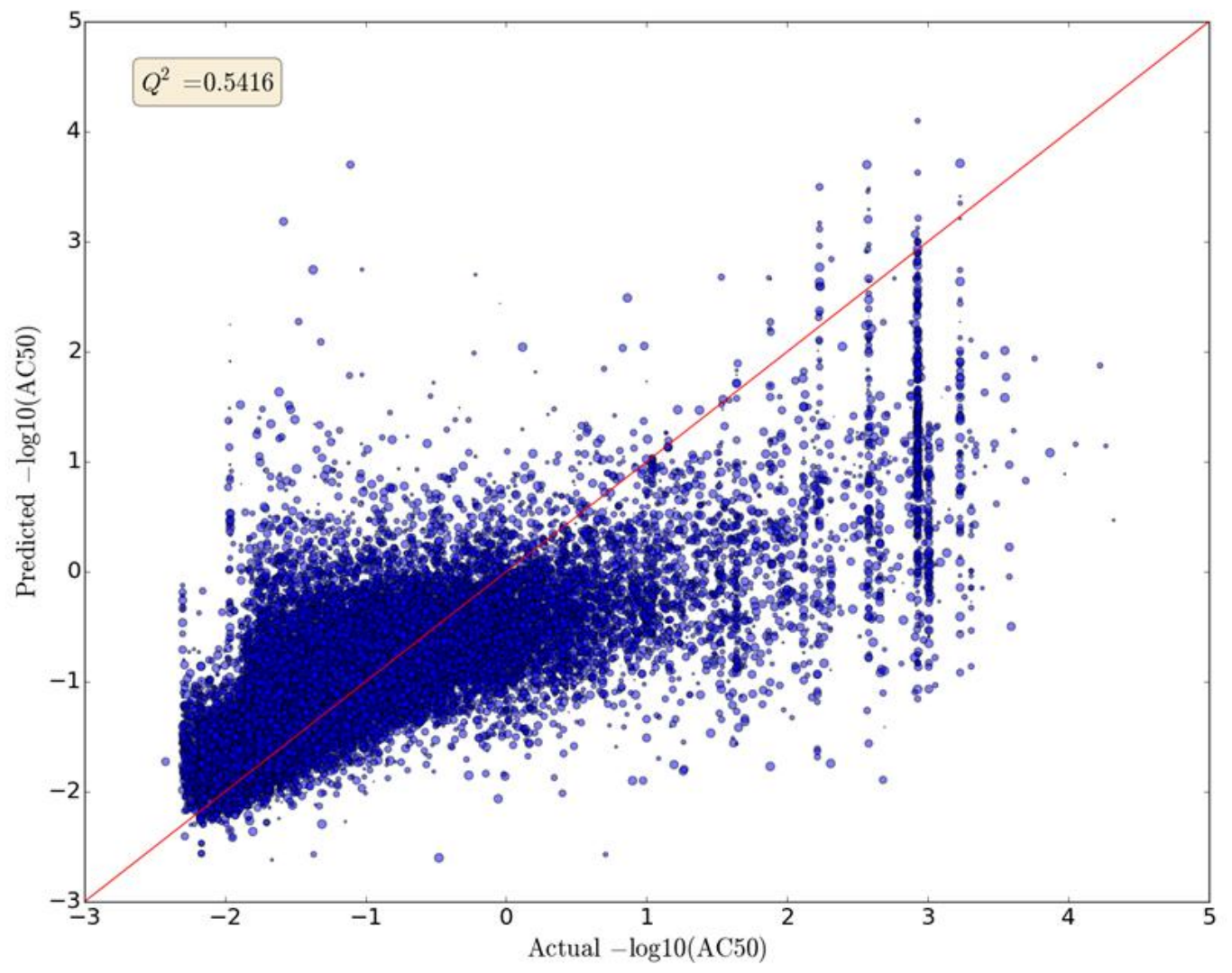

Figure 6 

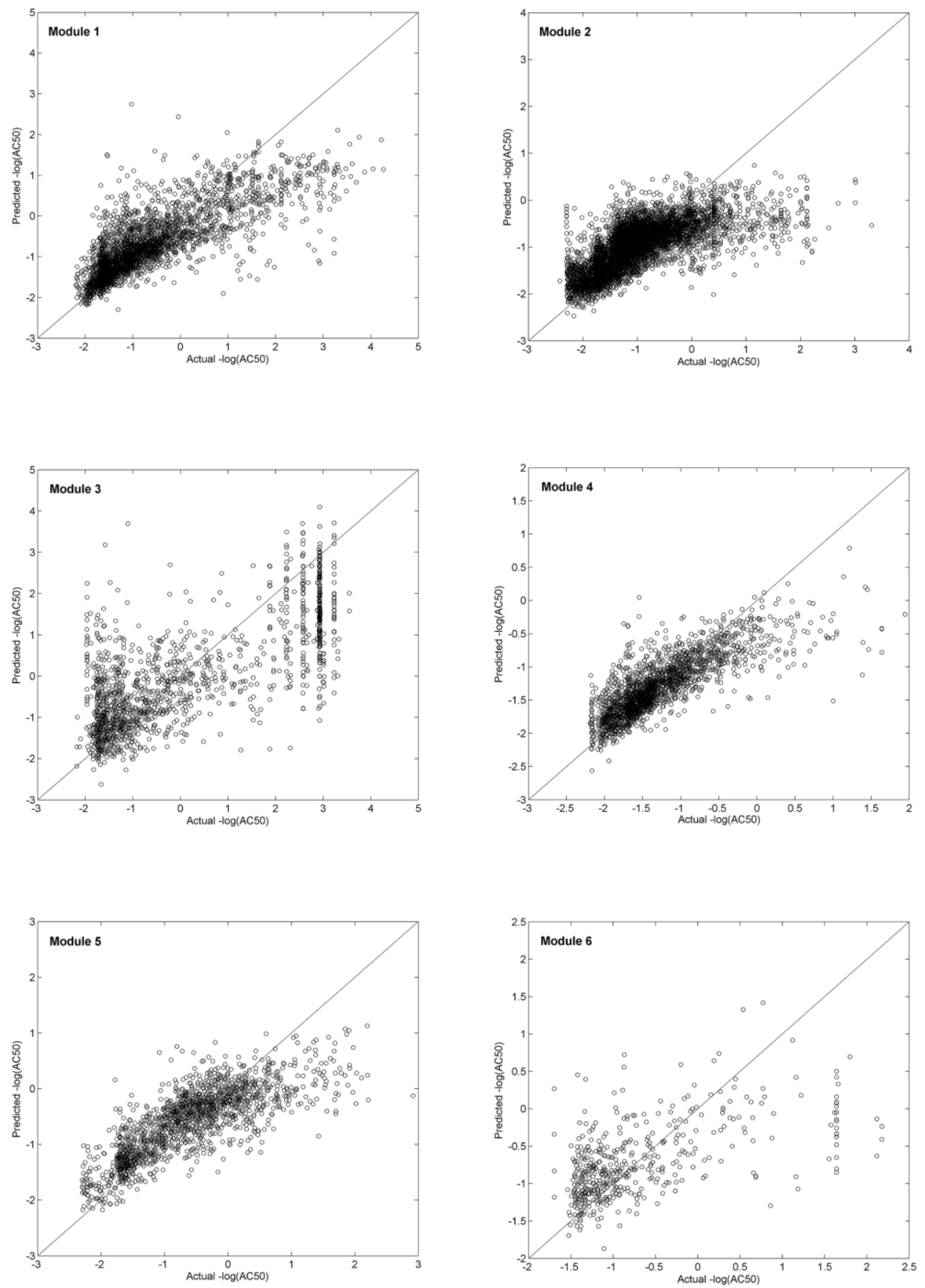

Figure 7 
A

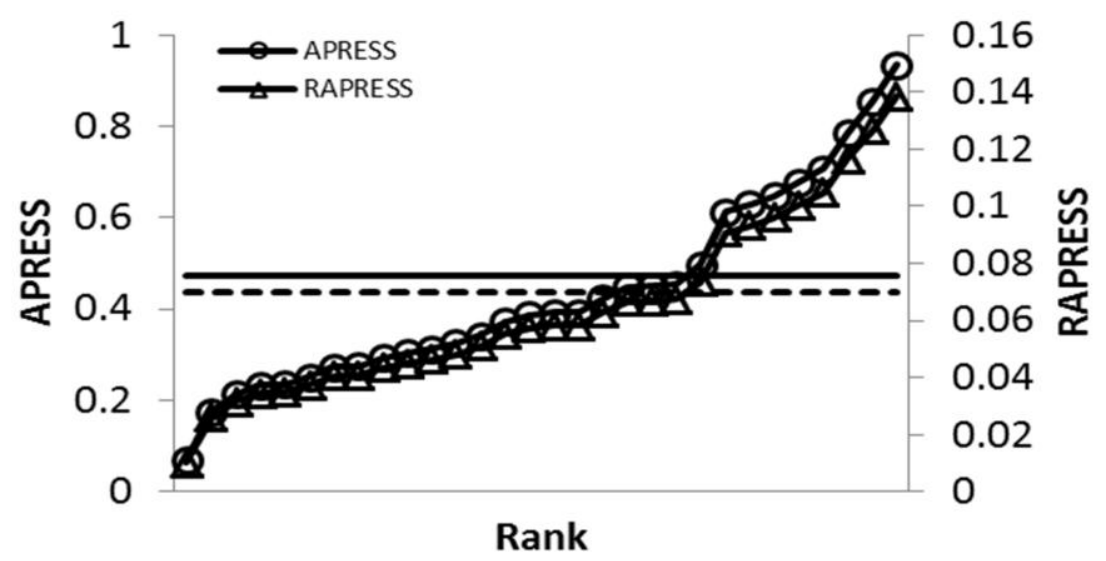

B

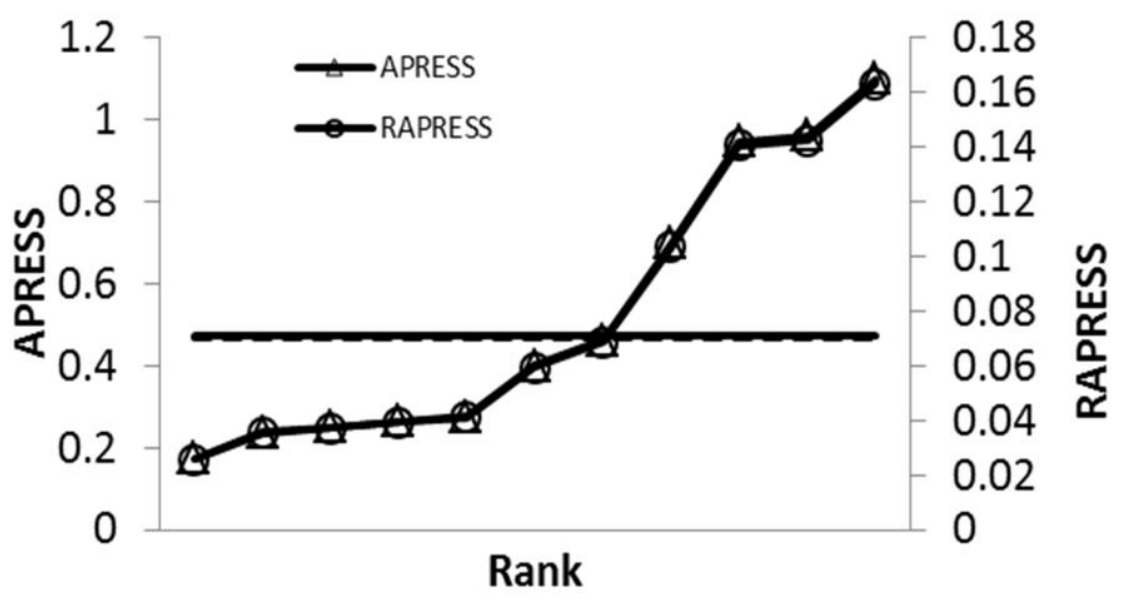

C

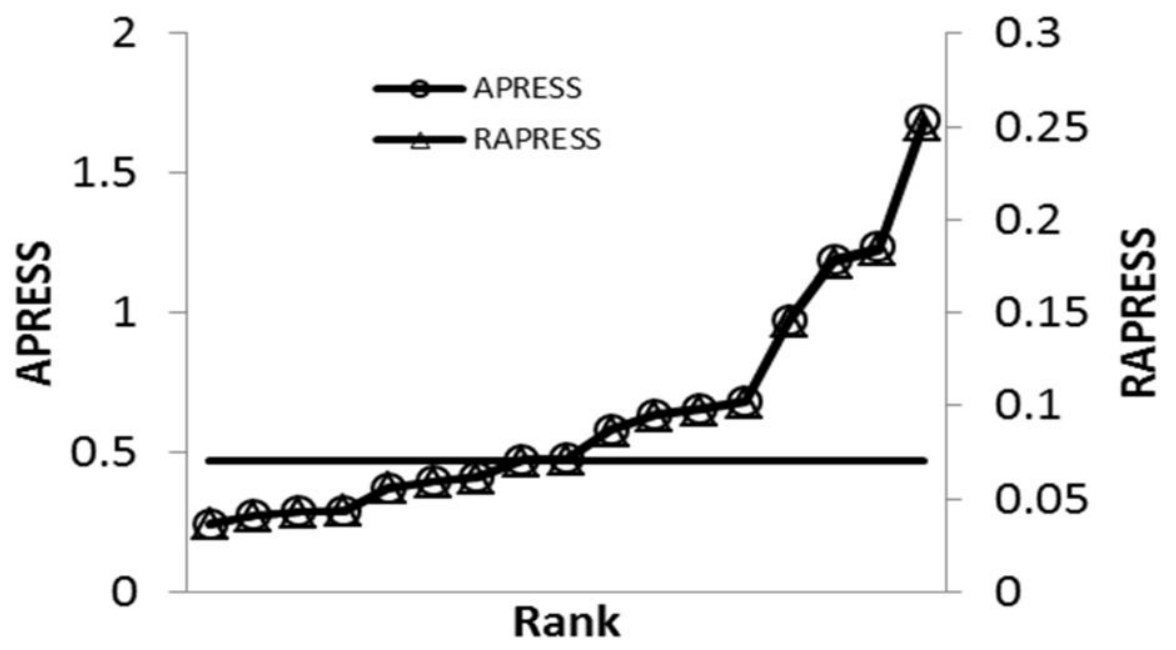

Figure 8 